\title{
Hormonal Imbalance in Polycystic Ovarian Syndrome (Pcos) in Teaching Hospitals in Ekiti State, Nigeria
}

\author{
0. G. Oyebanji*, M. F. Asaolu, E. O. Amonimo \\ Department of Biochemistry, Ekiti State University, Ado Ekiti, Nigeria \\ Email: *talktofisayo@yahoo.com
}

How to cite this paper: Oyebanji, O.G., Asaolu, M.F. and Amonimo, E.O. (2018) Hormonal Imbalance in Polycystic Ovarian Syndrome (Pcos) in Teaching Hospitals in Ekiti State, Nigeria. Open Journal of Obstetrics and Gynecology, 8, 1456-1464. https://doi.org/10.4236/ojog.2018.813147

Received: October 24, 2018

Accepted: November 25, 2018

Published: November 28, 2018

Copyright ( 92018 by authors and Scientific Research Publishing Inc. This work is licensed under the Creative Commons Attribution International License (CC BY 4.0).

http://creativecommons.org/licenses/by/4.0/

(c) (i) Open Access

\begin{abstract}
Polycystic ovary syndrome (PCOS) is one of the most common endocrine disorders of women at reproductive age and the major cause of anovulatory infertility. The aim of this study is to determine hormonal imbalance in women with PCOS in teaching hospitals in Ekiti State, Nigeria. Samples of 110 females (50 freshly diagnosed PCOS subjects and 60 healthy subjects) between the ages of 25 - 45 years old were collected from Ekiti State University Teaching Hospital, Ado Ekiti and Federal Teaching Hospital, Ido Ekiti, Ekiti State, Nigeria. Serum Luteinizing Hormone (LH), Follicle Stimulating Hormone (FSH), Total Testosterone, Prolactin, Progesterone, Estradiol and Insulin were evaluated in subjects' blood sample using Enzyme-Linked Immunosorbent assay (ELISA). Results obtained were subjected to statistical analysis $(\mathrm{p}<0.05)$. The PCOS subjects showed significant increase $(\mathrm{p}<0.05)$ in the concentrations of Total Testosterone, Insulin, LH, Estradiol and LH/FSH ratio, while FSH and progesterone concentrations showed significant decrease $(\mathrm{p}<0.05)$ in comparison with the healthy control group. However, there was no significant difference in the concentration of Prolactin when compared with the healthy control group. Also, the PCOS subjects' age at Menarche was significantly decreased $(\mathrm{p}<0.05)$ while their age showed no significant difference $(p>0.05)$ when compared with the healthy control group. Hormonal values obtained in this study indicates hormonal imbalance in PCOS. Further investigation into novel helpful markers is required as it might help in achieving the therapeutical target in PCOS management research.
\end{abstract}

\section{Keywords}

Polycystic Ovarian Syndrome, Insulin, Testosterone, Progesterone, Estradiol, Luteinizing Hormone 


\section{Introduction}

Polycystic ovary syndrome (PCOS) is one of the most common endocrine disorders of women at reproductive age and the major cause of anovulatory infertility [1] [2]. PCOS affects about $4 \%-12 \%$ of women, predominantly women of reproductive age with unclear etiologies [3]. This syndrome has the heterogenous collection of the signs and symptoms that gathered together to form a spectrum of disorder with the mild representation in some, whilst in others a severe disturbance of reproductive, endocrine and metabolic function [3]. PCOS is considered a multifactorial disorder with various genetic, endocrine and environmental abnormalities [4]. PCOS is not only based on the reproductive health issues, but metabolic and cardiovascular issues are also raised [5].

PCOS is a disease with high heterogeneity, and its clinical presentation mainly includes menstrual dysfunction, secondary amenorrhea, high androgen concentrations and ultrasound evidence of cysts within the ovary, hirsutism, acne, obesity and infertility [2] [6] [7]. The etiopathogenesis of PCOS remains elusive; it involves aberrant hormonal responses mediated by ovarian granulosa cells (GCs) during the progression of follicular development. In PCOS, high levels of androgenic hormones interfere with the hypothalamus-hypophysis axis, leading to an increase in the Luteinizing Hormone ( $\mathrm{LH}$ ), with the subsequent anovulation and amenorrea and therefore causing infertility [8]. Also, Insulin resistance has being reported a key pathophysiology of PCOS [9].

The long-term risk of PCOS includes a significantly increased risk of endometrial cancer, hyperlipidemia, obesity and non-insulin dependent diabetes mellitus (NIDDM), hypertension, cardiovascular disease, pregnancy-associated diabetes and hypertension [7] [10]. Wang et al. in 2001 reported a great risk of spontaneous abortion associated with PCOS compared with non-PCOS patients [11]. A high proportion of infertile female patients have PCOS with a wide range of possible symptoms [1]. The ethnicity and country of patients influences the extent of signs and symptoms in PCOS [12]. Incidence of this disease increases in our world today because of sedentary lifestyles, pollution, and excessive intake of junk food [13]. Lifestyle modification and weight reduction have been shown to decrease androgen effects, increase ovulation, and improve insulin sensitivity [14].

Treatment of PCOS is currently directed at symptoms rather than targeting a specific etiologic pathway, because the underlying pathophysiology is not fully determined [14]. Several treatment regimen extensively used for PCOS includes, metformin, cabergoline, myo-inositol, D-chiro-inositol, combined oral contraceptives including estradiol and non-androgenic progesterone, and aromatase inhibitors [15]. However, this research work estimates the levels of Insulin, Testosterone, Progesterone, Estradiol, Prolactin, Luteinizing hormone and Follicle stimulating hormone in women with Polycystic Ovarian syndrome and to detect the implication of its different concentrations in the management of the syndrome. 


\section{Materials and Methods}

\subsection{Subject Selection}

Freshly diagnosed women with Polycystic Ovarian Syndrome (PCOS) attending Ekiti State University Teaching Hospital, Ado Ekiti and Federal Teaching Hospital, Ido Ekiti, Ekiti State were used as the subject for this study. The population for the research project is 110 subjects; 60 relatively healthy subjects, 50 PCOS subjects with the ages of 25 - 45 years. The PCOS subjects were clinically diagnosed and confirmed according to Rotterdam Consensus Criteria [16]. Ethical approval was obtained from Ekiti State Teaching Hospital (EKSUTH) Ado Ekiti and Ekiti State University Ethics Committee. Letter of consent was issued to subjects with proper understanding of the research work before any data or sample is collected from them. Questionnaire was be used to collect all relevant information about the subjects' health history, socio-demographic data, anthropometric and life style habits.

\subsubsection{Subjects Inclusion Criteria}

1) Freshly diagnosed and physically examined PCOS subjects.

2) PCOS subjects aged 25 - 45 years old.

3) Relatively healthy subjects (subjects without PCOS) as control.

\subsubsection{Subjects Exclusion Criteria}

1) Healthy subjects without PCOS, currently on medication.

2) Unwilling PCOS subjects, not interested in being included in the research.

\subsection{Sample Collection}

Blood samples (10 mls) were collected intravenously 10 days after the regular menstrual cycleusing standard venipuncture technique in the entire subject into plain tubes. The collected blood samples were centrifuged at $3000 \mathrm{rpm}$ for 10 min using table top centrifuge (model 0508-1) to obtain the serum. The serum was carefully separated with the use of micro pipette. The samples were stored at temperatures of $-20^{\circ} \mathrm{C}$ for biochemical analysis.

\subsection{Biochemical Analysis}

\section{Determination of Hormones}

Determination of hormones; Luteinizing Hormone (LH), Follicle Stimulating Hormone (FSH), Insulin, Total testosterone, Androstenedione (androgen hormone), Prolactin, Progesterone, Estradiol and Cortisol in serum sample were carried out using the Enzyme-Linked Immunosorbent Assay (ELISA) as described by [17].

\section{Results}

Table 1 showed significant increase $(\mathrm{p}<0.05)$ in the concentrations of Insulin, Testosterone, Estradiol, LH and LH/FSH ratio in the PCOS subjects, while there was significant decrease $(\mathrm{p}<0.05)$ in the concentration of Progesterone and FSH 
Table 1. Levels of hormones in polycystic ovarian syndrome patients and control subjects.

\begin{tabular}{ccc}
\hline GROUP/PARAMETERS & CONTROL & PCOS \\
\hline INSULIN (mmol/L) & $3.13 \pm 0.44^{\mathrm{a}}$ & $7.89 \pm 1.31^{\mathrm{b}}$ \\
TESTOSTERONE (nmol/L) & $0.42 \pm 0.05^{\mathrm{a}}$ & $0.49 \pm 0.05^{\mathrm{b}}$ \\
PROLACTIN (mmol/L) & $12.05 \pm 1.33^{\mathrm{a}}$ & $13.81 \pm 0.77^{\mathrm{a}}$ \\
FSH (MIU/L) & $6.64 \pm 0.62^{\mathrm{a}}$ & $4.15 \pm 0.50^{\mathrm{b}}$ \\
LH (MIU/L) & $6.19 \pm 1.47^{\mathrm{a}}$ & $9.59 \pm 1.58^{\mathrm{b}}$ \\
(LH/FSH) & $1.32 \pm 0.24^{\mathrm{a}}$ & $2.38 \pm 0.59^{\mathrm{b}}$ \\
PROGESTERONE (ng/mL) & $7.38 \pm 1.28^{\mathrm{a}}$ & $4.91 \pm 1.20^{\mathrm{b}}$ \\
ESTRADIOL (pg/dL) & $136.67 \pm 8.40^{\mathrm{a}}$ & $163.57 \pm 9.20^{\mathrm{b}}$ \\
MENARCHE AGE (yrs) & $15.00 \pm 0.55^{\mathrm{a}}$ & $13.20 \pm 0.34^{\mathrm{b}}$ \\
AGE (yrs) & $34.82 \pm 2.17^{\mathrm{a}}$ & $33.70 \pm 1.47^{\mathrm{a}}$ \\
\hline
\end{tabular}

Values are presented as mean \pm SEM. Values with superscript $a$ and $b$ are significantly different at $p<0.05$ when compared with Non PCOS control group. PCOS = Polycystic Ovarian Syndrome; FSH = Follicle stimulating hormone; $\mathrm{LH}=$ Luteinizing hormone; $(\mathrm{LH} / \mathrm{FSH})=$ Ratio of $\mathrm{LH}$ and FSH.

in the PCOS subjects when compared with the control subjects. However, the level of Prolactin in PCOS showed no significant difference $(p>0.05)$ when compared with the control group.

Also, Table 1 showed significant increase $(\mathrm{p}<0.05)$ in the weight of PCOS subjects while there was significant decrease $(\mathrm{p}<0.05)$ in the menarche age of the PCOS subjects in comparison with the control subject. The age of the PCOS subjects showed no significant difference $(p>0.05)$ when compared with the control subject as shown in Table 1 .

Clinical Presentations

1) Ultrasound evidence of cysts within the ovary.

2) Menstrual dysfunction.

3) Infertility.

4) Amenorrhea.

5) High androgen concentrations.

6) Hirsutism/acne.

\section{Discussion}

Testosterone is the primary male sex hormone and an anabolic steroid. Testosterone is the primary male sex hormone and an anabolic steroid. High androgen levels also known as hyperandrogenemia is associated with menstrual cycle irregularities and adverse metabolic features, including insulin resistance, central obesity, dyslipidemia, and chronic inflammation in premenopausal women, which might lead to an increased cardiovascular risk. Nearly 80 percent of women with hyperandrogenism have polycystic ovaries [18]. There was significant increase $(\mathrm{p}<0.05)$ in the testosterone concentration of PCOS group when compared with Non-PCOS control group (Table 1). This study is in agreement 
with the work of Bartolone et al. (2000) who reported an extremely high level of Testosterone in PCOS [19]. This emphasizes the importance of testosterone in diagnosing of PCOS.

Insulin is a peptide hormone produced by beta cells of the pancreatic islets. It regulates the metabolism of carbohydrates, fats and protein by promoting the absorption of, especially, glucose from the blood into fat, liver and skeletal muscle cells [20]. When Glucose concentration in the blood is high, the pancreatic beta cells secrete insulin into the blood; when glucose levels are low, secretion of insulin is inhibited [21]. The concentration of Insulin in PCOS patient shows that there was significant increase at $\mathrm{p}<0.05$ when compared with the control subject (Table 1). This corresponds with the work of Meenakumari et al. (2004) who reported a high level of insulin level during luteal phase in the PCOS women [22]. Meenakumari et al. also suggested that increased insulin levels suppress progesterone production by granulosa cells collected from women with PCOS but not from normal women [22]. However, insulin may act at the pituitary level, the ovarian and/or the hepatic level to increase androgen synthesis and/or free testosterone serum concentrations. Meanwhile significant positive correlation between insulin and LH concentration during the luteal phase has also been reported [22].

Progesterone is an endogenous steroid and progestogen sex hormone involved in the menstrual cycle, pregnancy, and embryogenesis of humans and other species [23]. According to this study, there was significant decrease $(\mathrm{p}<0.05)$ in the progesterone level of PCOS subjects; this is in agreement with the work of Meenakumari et al. who reported low progesterone level during luteal phase in the PCOS women [23]. Hyperinsulinemia and/or insulin resistance was reported a possible cause of the Progesterone deficiency in PCOS [22]. High progesterone level means that ovulation did indeed occur and the egg was released from the ovary, but if the progesterone level is low the egg was probably not released leading to anovulation in PCOS [23].

Estradiol (E2), also spelled oestradiol, is a steroid, an estrogen, and the primary female sex hormone. It is named for and it's important in the regulation of the estrous and menstrual female reproductive cycles. The concentration of Estradiol in PCOS subjects in this study increased significantly in comparison with the control subject (Table 1). This correlates with the research work of Bartolone et al. who reported an extremely high level of Estradiol in PCOS, and suggested that the differential diagnosis of high levels of E2 +/- Testosterone should include PCOS [19].

Luteinizing hormone ( $\mathrm{LH}$ ) also known as lutropin is a hormone produced by gonadotropic cells in the anterior pituitary gland, it is crucial in regulating the function of ovaries in women. Also, LH helps to regulate the menstrual cycle and egg production (ovulation). However, there was significant increase $(\mathrm{p}<0.05)$ in the level of Luteinizing hormone in PCOS subjects compared with the control subjects. This study correlates with the research work of Yue et al. who reported an increase in the level of LH in PCOS group [24]. 
Follicle stimulating hormone (FSH) is a gonadotropin, a glycoprotein polypeptide hormone. FSH is synthesis and secreted by the gonadotropic cells of the anterior pituitary gland and regulates the development, growth, puberty maturation, and reproductive process of the body [25]. There was significant decrease $(p<0.05)$ in the concentration of FSH in the PCOS subjects compared with the control. The decrease in serum FSH level causes the smaller follicles in the current cohort to undergo atresia as they lack sufficient sensitivity to FSH to survive. Although an increase in $\mathrm{LH}$ level drives the ovarian theca cells to produce more androgens, insufficient follicle stimulating hormone (FSH) may be the more immediate cause of anovulation in PCOS patients [26]. Therefore, High level of LH and FSH deficiency may cause loss of menstrual cycles, infertility, decrease in sex drive and vaginal dryness and osteoporosis, which can result in a tendency to develop bone fractures and consequently lead to Polycystic Ovarian Syndrome.

Also, there was significant increase $(\mathrm{p}<0.05)$ in the level of LH/FSH ratio in PCOS subject compared with the control. This is in agreement with the work of Yue et al. and Malini and Roy George who reported that both LH and the LH/FSH ratio were strongly increased in the PCOS group. The increase in LH/FSH ratio indicates hormonal disorder in PCOS [24] [27].

Prolactin is a hormone whose primary function is to initiate lactation. It is released by the pituitary gland, a small organ located at the base of the brain that influences the entire body [28]. This study shows that prolactin has no significant difference $(\mathrm{p}>0.05)$ when compared with control subject (Table 1). High level of prolactin has been shown not to be associated with the PCOS [29]. Szosland et al. also stated that hyperprolactinemia does not seem to be more frequent in PCOS women and it might not be considered as characteristic feature of PCOS [29].

Menarche is the first menstrual cycle, or first menstrual bleeding, in female humans. From both social and medical perspectives, it is often considered the central event of female puberty, as it signals the possibility of fertility. Girls experience menarche at different ages. The timing of menarche is influenced by female biology, as well as genetic and environmental factors, especially nutritional factors. This study showed significant decrease $(\mathrm{p}<0.05)$ in menarche age of PCOS subjects in comparison with Non-PCOS subjects. This is in agreement with the work of Carroll et al. who reported that women with PCOS have an earlier age at pubarche on average than control subjects, influenced not only by weight but also by genetic variants [30]. However, there was no significant difference $(\mathrm{p}<0.05)$ in the age of PCOS group when compared with Non-PCOS group. Therefore, age at menarche might be helpful in early detection of PCOS in Females.

\section{Conclusions}

PCOS is a heterogeneous disorder of uncertain cause. In PCOS women, normal 
gonadotropin-ovarian axis is disturbed; this is reflected by the hormonal disorders. The results obtained in this study showed that testosterone, insulin, progesterone, estradiol, FSH, and LH were implicated in PCOS subjects. Therefore, this study suggests an interrelationship between insulin, LH and testosterone in promoting the hormonal disorders in PCOS subjects. Improving insulin sensitivity by using insulin-sensitizing agents might help decrease circulating androgen levels, improve ovulation rate, and improve glucose tolerance in PCOS [31].

Regardless of bodily features/weight, highest priority should be placed on regular checkup for adolescent females. However factors that could be associated with insulin resistance and hyperinsulinemia should be monitored in Adolescent girls. Also the age at menarche of adolescents should not be overlooked as it might help in early diagnosis of PCOS, hence aids the management and treatment of PCOS. An efficient and timely identification, management and treatment will go a long way in reducing the risks posed by Polycystic Ovarian Syndrome. More research is suggested to be done to explore novel biomarkers for early diagnosis of PCOS.

\section{Acknowledgements}

All the Professors in the department of Biochemistry, Ekiti state university, Nigeria and the chief technologists of the university are highly appreciated for their contributions that made this research work a success. Also, all the subjects that participated in this research study are appreciated for their consent.

\section{Funding}

This research did not receive any specific grant from any funding agency in the public, commercial or not-for-profit sector.

\section{Authors Contribution}

O.O.G and A.M.F conceived and designed the research study. O.O.G, A.M.F and A.E.O performed the experiment and data collection. O.O.G carried out data analysis and interpretation with support from A.M.F and A.E.O. O.O.G wrote the manuscript with support from A.M.F. and A.E.O. O.O.G, A.M.F and A.E.O revised the manuscript critically. O.O.G, A.M.F and A.E.O made the final approval of the manuscript to be published.

\section{Conflicts of Interest}

The authors declare that there is no conflict of interests regarding the publication of this research work.

\section{References}

[1] Joham, A.E., Teede, H.J., Ranasinha, S., Zoungas, S. and Boyle, J. (2015) Prevalence of Infertility and Use of Fertility Treatment in Women with Polycystic Ovary Syndrome: Data from a Large Community-Based Cohort Study. Journal of Women's 
Health, 24, 299-307. https://doi.org/10.1089/jwh.2014.5000

[2] Zuo, T., Zhu, M. and Xu, W. (2016) Roles of Oxidative Stress in Polycystic Ovary Syndrome and Cancers. Oxidative Medicine and Cellular Longevity, 2016, Article ID: 8589318. https://doi.org/10.1155/2016/8589318

[3] Bhuvaneshwari, S., Poornima, R. and Averal, H.I. (2015) Detection of Polycystic Ovary Syndrome and Its Treatment with Pergulariadaemia in Rat Models. IOSR Journal of Pharmacy, 5, 42-49.

[4] De Leo, V., Musacchio, M.C., Cappelli, V., Massaro, M.G., Morgante, G. and Petraglia, F. (2016) Genetic, Hormonal and Metabolic Aspects of PCOS: An Update. Reproductive Biology and Endocrinology, 14, 38. https://doi.org/10.1186/s12958-016-0173-x

[5] King, J. (2006) Polycystic Ovary Syndrome. Journal of Midwifery and Women's Health, 51, 415. https://doi.org/10.1016/j.jmwh.2006.01.008

[6] Schmid, J., Kirchengast, S., Binstorfer, E.V. and Huber, J. (2004) Infertility Caused by PCOS-Health-Related Quality of Life among Austrian and Moslem Immigrant Women in Austria. Human Reproduction, 19, 2251-2257. https://doi.org/10.1093/humrep/deh432

[7] Norman, R.J., Masters, L., Milner, C.R., Wang, J.X. and Davies, M.J. (2001) Relative Risk of Conversion from Normoglycaemia to Impaired Glucose Tolerance or Non-Insulin Dependent Diabetes Mellitus in Polycystic Ovarian Syndrome. Human Reproduction, 16, 1995-1998. https://doi.org/10.1093/humrep/16.9.1995

[8] Farrell, K. and Antoni, M.H. (2010) Insulin Resistance, Obesity, Inflammation, and Depression in Polycystic Ovary Syndrome: Biobehavioral Mechanisms and Interventions. Fertility and Sterility, 79, 956-962.

https://doi.org/10.1016/j.fertnstert.2010.03.081

[9] Jin, J.K. and Young, M.C. (2013) Dyslipidemia in Women with Polycystic Ovary Syndrome. Obstetrics and Gynecology Science, 56, 137-142. https://doi.org/10.5468/ogs.2013.56.3.137

[10] Solomon, C.G. (1999) The Epidemiology of Polycystic Ovary Syndrome: Prevalence and Associated Disease Risks. Endocrinology and Metabolism Clinics of North America, 28, 247-263. https://doi.org/10.1016/S0889-8529(05)70069-4

[11] Wang, J.X., Davies, M.J. and Norman, R.J. (2001) Polycystic Ovarian Syndrome and the Risk of Spontaneous Abortion Following Assisted Reproductive Technology treatment. Human Reproduction, 16, 2606-2609. https://doi.org/10.1093/humrep/16.12.2606

[12] Alili, A. and Idrizi, E.A. (2014) Clinical and Biochemical Characteristics in Women with Polycystic Ovarian Syndrome in the Region of Pollog, Republic of Macedonia. European Scientific Journal, 10, 179-185.

[13] Khot, B.M., Lad, M.D., Patil, A.J. and Kakad, A.C. (2015). Clinical Efficacy of Ayurveda Treatment on Polycystic Ovarian Syndrome. IOSR Journal of Pharmacy, 5, 42-49.

[14] National Institutes of Health (NIH) (2012) Evidence-Based Methodology Workshop on Polycystic Ovary Syndrome: Final Report. National Institutes of Health, Bethesda, 1-14.

[15] Swaroop, A., Jaipuriar, A.S., Gupta, S.K., Bagchi, M., Kumar, P., Preuss, H.G. and Bagchi, D. (2015) Efficacy of a Novel Fenugreek Seed Extract (Trigonella foenum-graecum, Furocyst ${ }^{\mathrm{TM}}$ ) in Polycystic Ovary Syndrome (PCOS). International Journal of Medical Science, 12, 825-831. https://doi.org/10.7150/ijms.13024 
[16] The Rotterdam ESHRE/ASRM-Sponsored PCOS Consensus Workshop Group (2004) Revised 2003 Consensus on Diagnostic Criteria and Long-Term Health Risks Related to Polycystic Ovary Syndrome (PCOS). Human Reproduction, 19, 41-47.

[17] Carl, A.B., Edward, R.A. and David, E.B. (2012) Tietz Textbook of Clinical Chemistry and Molecular Diagnostics. 5th Edition, Elsevier Inc., Saunders, 1-60.

[18] Marilyn, R. and Richardson, M.D. (2003) Current Perspectives in Polycystic Ovary Syndrome. American Family Physician, 68, 697-705.

[19] Bartolone, L., Smedile, G., Arcoraci, V., Trimarchi, F. and Benvenga, S. (2000) Extremely High Levels of Estradiol and Testosterone in a Case of Polycystic Ovarian Syndrome. Hormone and Clinical c Similarities with the Phenotype of the Alpha Estrogen Receptor Null Mice. Journal of Endocrinology, 23, 467-472.

[20] Stryer, L. (2013) Biochemistry. W.H. Freeman and Company, New York, 773-774.

[21] Koeslag, J.H., Saunders, P.T. and Terblanche, E. (2003) A Reappraisal of the Blood Glucose Homeostat Which Comprehensively Explains the Type 2 Diabetes Mellitus-Syndrome X Complex. The Journal of Physiology, 549, 333-546. https://doi.org/10.1113/jphysiol.2002.037895

[22] Meenakumari, K.J., Agarwal, S., Krishna, A. and Pandey, L.K. (2004) Effects of Metformin Treatment on Luteal Phase Progesterone Concentration in Polycystic Ovary Syndrome. Brazilian Journal of Medical and Biological Research, 37, 1637-1644. https://doi.org/10.1590/S0100-879X2004001100007

[23] King, T.L. and Brucker, M.C. (2010) Pharmacology for Women's Health. 2nd Edition, Jones and Bartlett Publishers, Burlington, 372-373.

[24] Yue, L., Shi, G.Z., Gang, L., Chi, K.L., Zhi, Q.X., Xian, W.S., Jin, L.M., Wai, Y.C. and Hong, B.L. (2017) Identification of Reference Genes for qRT-PCR in Granulosa Cells of Healthy Women and Polycystic Ovarian Syndrome Patients. Scientific Reports, 7, Article No. 6961.

[25] Matthew, P.R., Rose, E.G. and Adam, H.B. (2000) Definition and Measurement of Follicle Stimulating Hormone. Endocrine Reviews, 21, 5-22.

https://doi.org/10.1210/edrv.21.1.0388

[26] Sampurna, K., Reddy, B.V., Vijayaraghavan, R. and Rajesh, P. (2017) Role of Steroid Hormones in Polycystic Ovarian Syndrome (PCOS) in South Indian Women. International Journal of Research in Ayurveda and Pharmacy, 8, 234-237. https://doi.org/10.7897/2277-4343.082118

[27] Malini, N.A. and Roy-George, K. (2017) Evaluation of Different Ranges of LH: FSH Ratios in Polycystic Ovarian Syndrome (PCOS)—Clinical Based Case Control Study. General and Comparative Endocrinology, 260, 51-57. https://doi.org/10.1016/j.ygcen.2017.12.007

[28] Mancini, T., Casanueva, F.F. and Giustina, A. (2008) Hyperprolactinemia and Prolactinomas. Endocrinology and Metabolism Clinics of North America, 37, 67-99. https://doi.org/10.1016/j.ecl.2007.10.013

[29] Szosland, K., Pawlowicz, P. and Lewiński, A. (2015) Prolactin Secretion in Polycystic Ovary Syndrome (PCOS). Neuro Endocrinology Letters, 36, 53-58.

[30] Carroll, J., Saxena, R. and Welt, C.K. (2012) Environmental and Genetic Factors Influence Age at Menarche in Women with Polycystic Ovary Syndrome. Journal of Pediatric Endocrinology and Metabolism, 25, 459-466. https://doi.org/10.1515/jpem-2012-0047

[31] American College of Obstetricians and Gynecologists (ACOG) (2018) ACOG Practice Bulletin No. 194: Polycystic Ovary Syndrome. Obstetrics and Gynecology, 131, e157-e171. https://doi.org/10.1097/AOG.0000000000002656 